\title{
Manipulating coincident charge and spin order with pressure and field in a doped manganite
}

\author{
A. S. Roy, A. Husmann, and T. F. Rosenbaum \\ The James Franck Institute and Department of Physics, The University of Chicago, Chicago, Illinois 60637 \\ J. F. Mitchell \\ Materials Science Division, Argonne National Laboratory, Argonne, Illinois 60439
}

(Received 17 November 2000; published 9 February 2001)

\begin{abstract}
The onset of charge order occurs simultaneously with the transition from ferromagnetic metal to antiferromagnetic insulator in $\mathrm{Nd}_{0.5} \mathrm{Sr}_{0.5} \mathrm{MnO}_{3}$. The application of hydrostatic pressures $P \geqslant 15 \mathrm{kbar}$ removes the degeneracy, as recorded in both the electrical resistivity and the magnetic susceptibility. The charge-ordered state moves swiftly to higher temperature with increasing pressure at the expense of the ferromagnetic charge liquid, dominating the energetics. An applied magnetic field $H$ suppresses both the pressure-split charge order and the antiferromagnetism, but charge order preferentially, so by $H=2 \mathrm{~T}$ the two transitions are reunited. Finally, depinning domains of charge order with electric field deep in the antiferromagnetic insulator permits us to monitor pressure-induced changes in the charge (poly)crystal.
\end{abstract}

DOI: 10.1103/PhysRevB.63.094416 PACS number(s): 75.30.Kz, 71.27.+a, 71.30.+h, 71.45.Lr

The perovskite manganites harbor an assortment of competing instabilities. It is their magnetic-field driven metalinsulator transition that provides the colossal magnetoresistance of prominent note, ${ }^{1}$ but charge localization is only the first part of the story. Ferromagnetic and antiferromagnetic transitions, orbital order, charge crystallization, and structural distortions all can arise in close vicinity to the metalinsulator transition. ${ }^{2}$ The competition between different states manifests itself in rich and varied phase diagrams across the mixed-valent manganite spectrum and in a tendency toward microphase separation. ${ }^{3}$

At 50\% hole density, the interplay between the charge, spin, orbital, and structural degrees of freedom can be particularly complex. ${ }^{4,5}$ In $\mathrm{Nd}_{0.5} \mathrm{Sr}_{0.5} \mathrm{MnO}_{3}$, there is a first-order transition with decreasing temperature from ferromagnetic charge liquid to charge ordered, antiferromagnetic insulator. Upon the application of magnetic field, the metal-insulator transition can be driven to zero temperature, with a drastic expansion of the hysteresis width. ${ }^{6}$ The fundamental origin of the charge ordering and its relationship to the accompanying magnetic and strain relieving transitions-which is the horse and which is the cart-is not known. ${ }^{7}$

$\mathrm{Nd}_{0.5} \mathrm{Sr}_{0.5} \mathrm{MnO}_{3}$ belongs to the broader class of materials with competing order parameters. Most notable are the heavy fermion compounds with linked magnetic and superconducting order. Theoretical descriptions of the multiple superconducting transitions in $\mathrm{UPt}_{3}$ and $(\mathrm{U}, \mathrm{Th}) \mathrm{Be}_{13}$ have been aided by the ability to manipulate the occurrence and number of phases using field, stress, and pressure. ${ }^{8}$ We apply this experimental approach to the coincident antiferromagnetic/ ferromagnetic, charge order/disorder transitions in $\mathrm{Nd}_{0.5} \mathrm{Sr}_{0.5} \mathrm{MnO}_{3}$. We split the two transitions with hydrostatic pressure, and find that the charge ordered state is thermally more robust. A magnetic field can merge the transitions once again, but affects the spin and charge instabilities in a different manner from pressure.

Twinned single crystals of $\mathrm{Nd}_{0.5} \mathrm{Sr}_{0.5} \mathrm{MnO}_{3}$ were melt grown in oxygen at 2 bars using a floating zone arc image furnace equipped with double hemiellipsoidal mirrors, ${ }^{9}$ and subsequently annealed in flowing oxygen. Samples of typical dimensions $(4 \times 1 \times 1) \mathrm{mm}^{3}$ were cut from a many-cm-long boule and electrical contacts were attached using silver paint covered by silver epoxy for structural stability. Crystals cut from all parts of the boule exhibited sharp, ambient pressure transitions from paramagnetic metal to ferromagnetic metal at $T_{C}=254 \pm 1 \mathrm{~K}$ and from ferromagnetic metal to antiferromagnetic insulator at $T_{\mathrm{MI}}=159 \pm 1 \mathrm{~K}$. The fact that the resistivity at ambient pressure in the metal is as low as previously reported data ${ }^{6}$ and even higher after the transition into the insulator testifies to the superior quality of the crystals. We applied hydrostatic pressures $P$ up to 21 kbar using a $\mathrm{BeCu}$ piston-anvil self-locking pressure clamp with a WC insert. The sample with four electrical leads and a secondary coil wrapped around it for simultaneous measurements of the magnetic susceptibility, as well as a piece of high-purity $\mathrm{Pb}$ for use as a manometer, were sealed in the Teflon bucket using Fluorinert as the pressure medium. The pressure cell with a primary coil wrapped around it was then mounted in a helium cryostat centered in an 8-T superconducting magnet. Ohmic and frequency-independent measurements of the resistivity $\rho(T, P, H)$ were obtained using a standard lock-in technique. We characterized the charge ordered state through its nonlinear response at liquid-helium temperature, using square-wave excitation to prevent rounding at the threshold electric field for non-Ohmicity $E_{\mathrm{th}}$. Although $\rho(T=4.2 \mathrm{~K})$ changed by orders of magnitude with $P, E_{\text {th }}$ changed relatively little, indicating that the nonlinear $I-V$ characteristic cannot be attributed to $I^{2} R$ heating.

The primary effect of pressures $P<15$ kbar is to depress the metal-insulator transition temperature. ${ }^{10}$ We plot in Figs. 1(a) and 1(b) the electrical resistivity $\rho(T)$ and the magnetic susceptibility $\chi(T)$ on cooling at $P=10 \mathrm{kbar}$. The sharp rise in the resistivity of more than three orders of magnitude at $T=155 \pm 1 \mathrm{~K}$ marks the transition into the insulating state. It is accompanied by an equally sharp drop in the susceptibility, indicating the bulk nature of both signatures. A narrow cusp in $\chi(T)$ and a subtle inflection in $\rho(T)$ designate the transition from paramagnetic metal to ferromagnetic metal at $T_{C}=256 \pm 1 \mathrm{~K}$. 

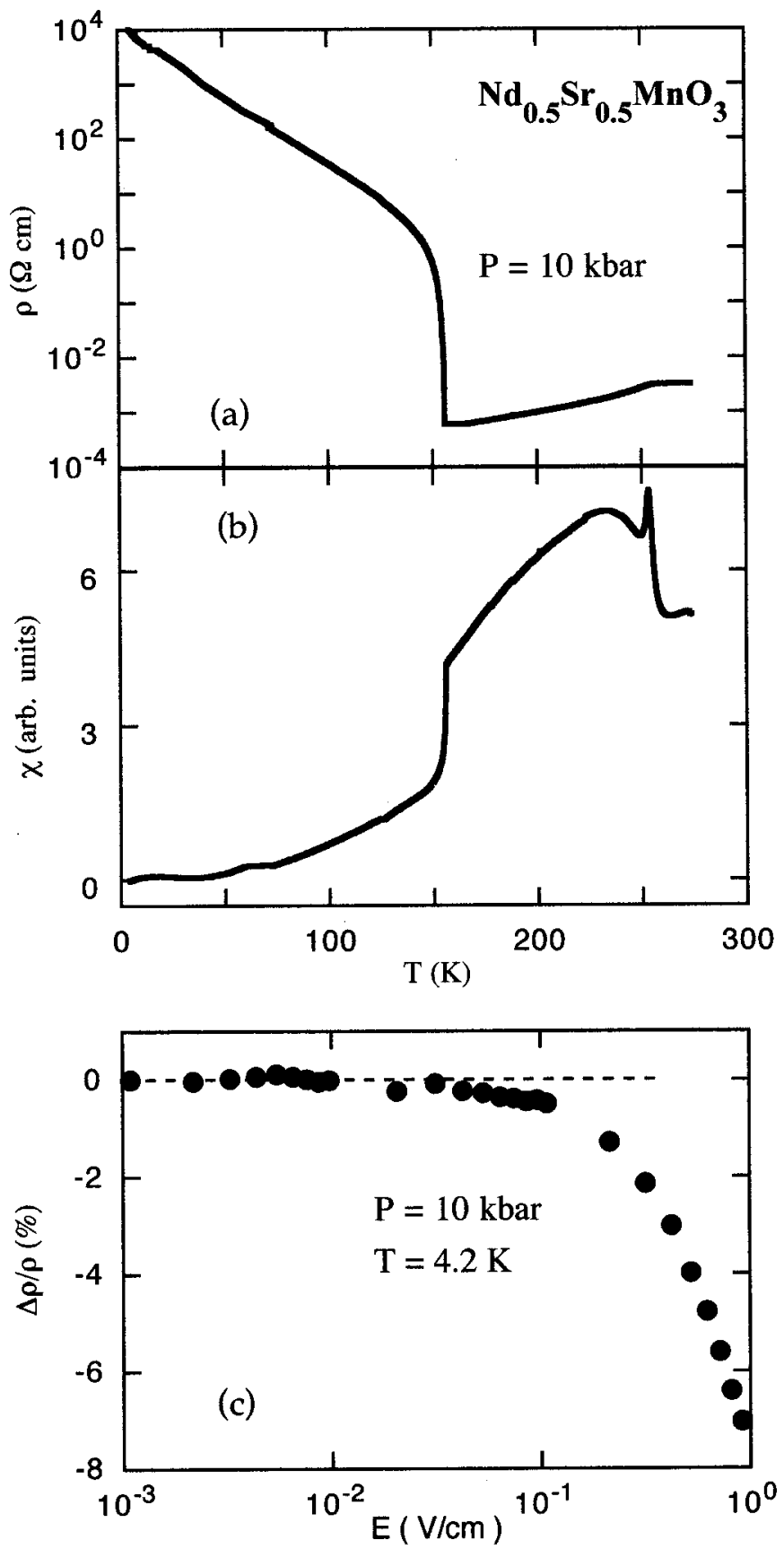

FIG. 1. (a) Electrical resistivity $\rho$ and (b) magnetic susceptibility $\chi$ as temperature $T$ is decreased at pressure $P=10 \mathrm{kbar}$. The inflection in $\rho$ and cusp in $\chi$ at $T_{C}=256 \mathrm{~K}$ marks the transition form paramagnetic to ferromagnetic metal; the sharp rise in $\rho$ at $T_{\mathrm{MI}}$ $=155 \mathrm{~K}$ marks the transition into the antiferromagnetic insulator, with charge order signaled by the sharp drop in $\chi$. (c) Deep in the insulator, the nonlinear behavior of the resistivity above a threshold electric field $E_{\mathrm{th}} \sim 0.06 \mathrm{~V} / \mathrm{cm}$ can be attributed to sliding domains of charge order of many microns extent (see text).

The sharp drop in $\chi$ at $T=155.5 \pm 0.5 \mathrm{~K}$ rides on a more gradual reduction in the susceptibility over an extended temperature range. Neutron-scattering, magnetic-susceptibility, and electron-diffraction measurements of the related perovskite manganite $(\mathrm{Bi}, \mathrm{Ca}) \mathrm{MnO}_{3}$ show that the sharp drop in $\chi(T)$ is associated with the actual charge ordering transition,

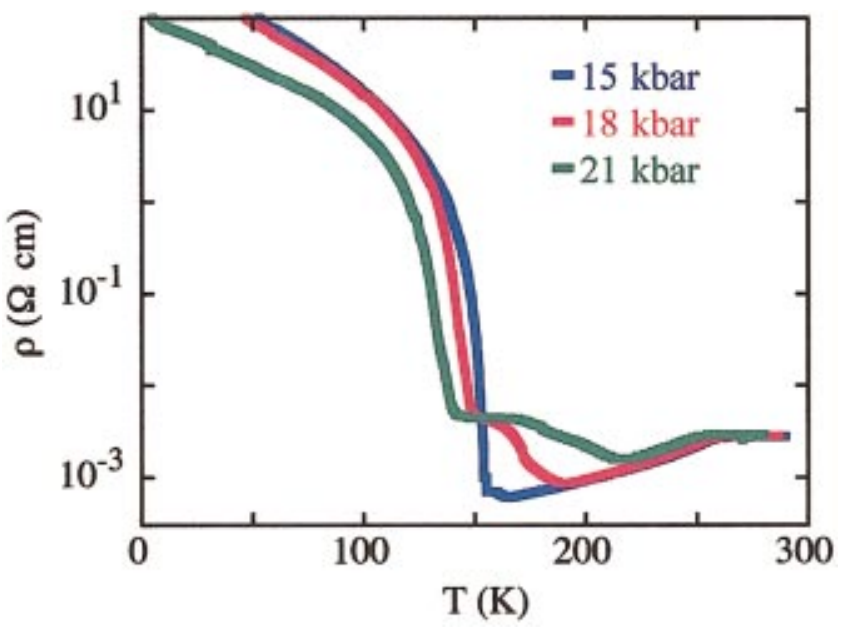

FIG. 2. (Color) Pressures $P \geqslant 15$ kbar can split the coincident antiferromagnetic insulator and charge order transitions.

while the further reduction in the magnetic susceptibility results from the replacement of ferromagnetic spin fluctuations by antiferromagnetic spin fluctuations. ${ }^{11}$ Deep in the antiferromagnetic insulator, it is possible to study the extent of charge order through the electric-field dependence of the resistivity [Fig. $1(\mathrm{c})]$. Above $E_{\mathrm{th}} \sim 0.06 \mathrm{~V} / \mathrm{cm}$ (corresponding to a power level $\sim 10^{-10} \mathrm{~W}$ ), there is an increase in the conductivity that we attribute to depinned, sliding regions of charge order. By analogy to charge-density wave systems, we can estimate the correlation length $\xi$ of the charge order from $E_{\text {th }}$ in the limit of weak pinning: ${ }^{12} E_{\mathrm{th}} \approx A\left(K / \rho R_{o}\right)\left(R_{o} / \xi\right)^{2}$, where $A$ is a constant of order unity and the shear modulus $K \sim e^{2} R_{o}{ }^{-4} \varepsilon_{\infty}{ }^{-1}$. Here, $R_{o}$ is the lattice spacing of the electron order, $k_{F}^{-1} \approx 11 \AA$, and $\varepsilon_{\infty} \sim 10$ is the high-frequency dielectric constant. Taking $A=1$, we find $\xi \approx 4.5 \mu \mathrm{m}$, indicating consistent charge order over thousands of lattice spacings.

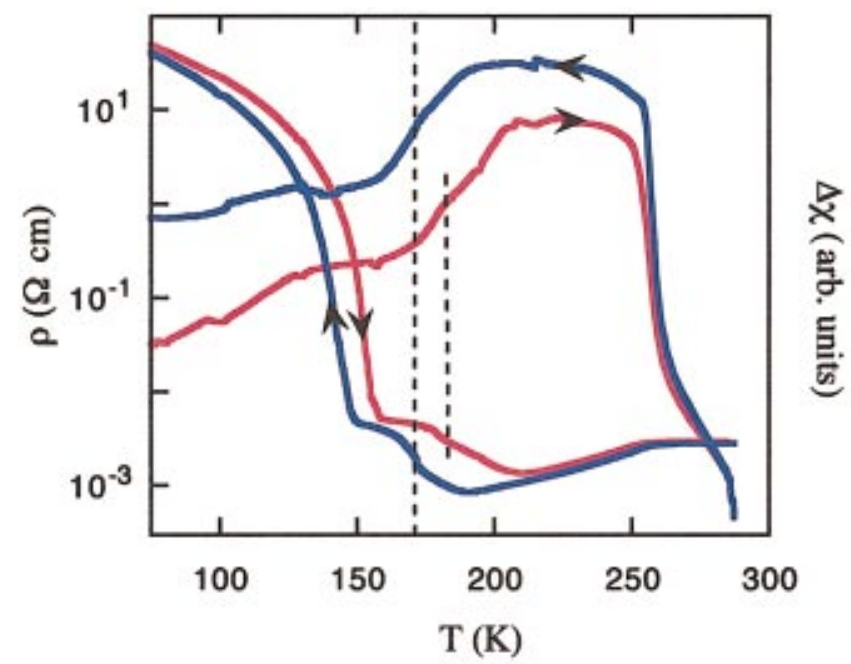

FIG. 3. (Color) Resistivity $\rho$ and susceptibility $\chi$ on both cooling (blue) and warming (red) at $P=18 \mathrm{kbar}$. The pronounced drop in $\chi(T)$, signaling charge order, tracks the upper transition in $\rho(T)$ (dotted lines). $\Delta \chi$ is $\chi(T)$ less its background linear temperature variation for $75<T<225 \mathrm{~K}$. 


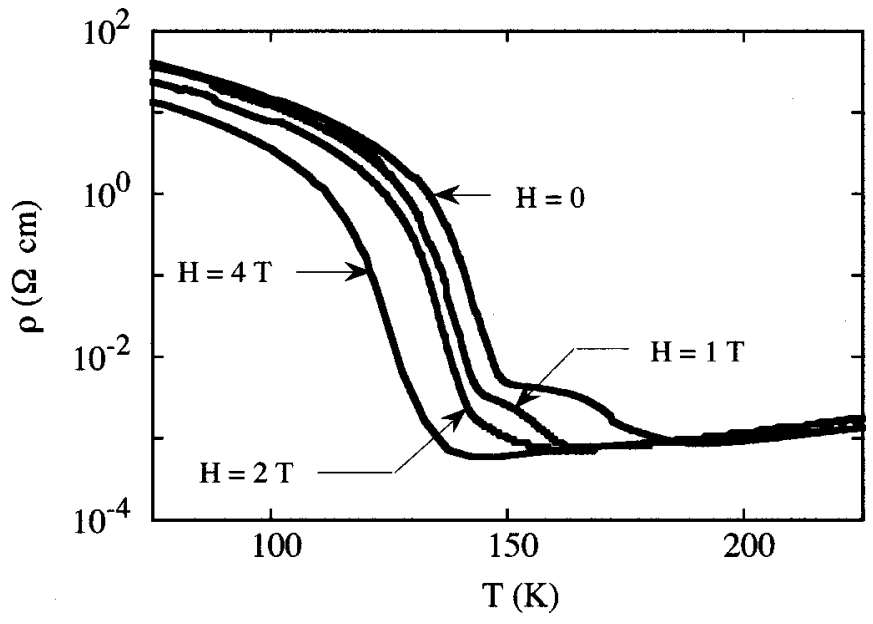

FIG. 4. A magnetic field $H<2 \mathrm{~T}$ can merge the pressure-split charge and spin transitions at $P=18$ kbar.

At pressures $P \geqslant 15$ kbars, the coincident charge and spin transitions split apart. We illustrate this process in Fig. 2. The ferromagnetic metal remains essentially undisturbed, but the rise in $\rho(T)$ now occurs in two unequal episodes that separate with increasing $P$. The initial, smaller, and broader rise moves to higher $T$ as $P$ is ratcheted up from 15 to 21 kbars, while the sharp transition into the insulator continues to be depressed in temperature. The magnetic susceptibility permits the identification of the phases. We superpose in Fig. $3 \rho(T)$ and $\Delta \chi(T)$ for both cooling and warming at $P$ $=18 \mathrm{kbar}$. For the sake of clear comparison, we have subtracted the smooth, linear depression in $\chi(T)$ for $75<T$ $<225 \mathrm{~K}$ [cf. Fig. 1(b)] to obtain $\Delta \chi(T)$. The pronounced drop in the susceptibility, shown to result from the chargeordering transition, tracks the upper feature in $\rho(T)$ (dotted lines). Hence pressure forces the antiferromagnetic transition below the charge ordering transition in $\mathrm{Nd}_{0.5} \mathrm{Sr}_{0.5} \mathrm{MnO}_{3}$, akin to the phase sequence observed in ambient pressure $\mathrm{La}_{1-x} \mathrm{Ca}_{x} \mathrm{MnO}_{3}$ for $x>0.5{ }^{13}$

The fact that charge order encroaches on the hightemperature ferromagnetic state is consistent with the underlying transport mechanism: ferromagnetic double exchange and its associated metallic conduction should diminish as charge orders. Differential volume effects in $\mathrm{Nd}_{0.5} \mathrm{Sr}_{0.5} \mathrm{MnO}_{3}$ also support a $d T_{\mathrm{CO}} / d P>0$. High-resolution crystallographic data indicate that the volume of the charge ordered phase is a few hundredths of a percent smaller than that of the ferromagnetic phase. ${ }^{14,15}$

An applied field $H \sim 14 \mathrm{~T}$ will reduce the $P=0$ metalinsulator transition temperature to zero. ${ }^{16} \mathrm{We}$ demonstrate in Fig. 4 that increasing the magnetic field at $P=18 \mathrm{kbar}$ lowers both the charge ordering and antiferromagnetic transition temperatures, but in such a way that they merge by $H$ $=2 \mathrm{~T}$. The pressure-split charge order, with $d T_{\mathrm{CO}} / d H$ $\sim 16 \mathrm{~K} / \mathrm{T}$ for $0<H<2 \mathrm{~T}$, is four times more sensitive to field than the antiferromagnetic transition with slope $d T_{\mathrm{AF}} / d H \sim 4 \mathrm{~K} / \mathrm{T}$. It is $T_{\mathrm{AF}}(H)$, however, that appears to set the scale for the further evolution of the reunited charge and spin transitions: $d T_{\mathrm{MI}} / d H \sim 5 \mathrm{~K} / \mathrm{T}$ for $2<H<8 \mathrm{~T}$.

We summarize our pressure and magnetic field results in
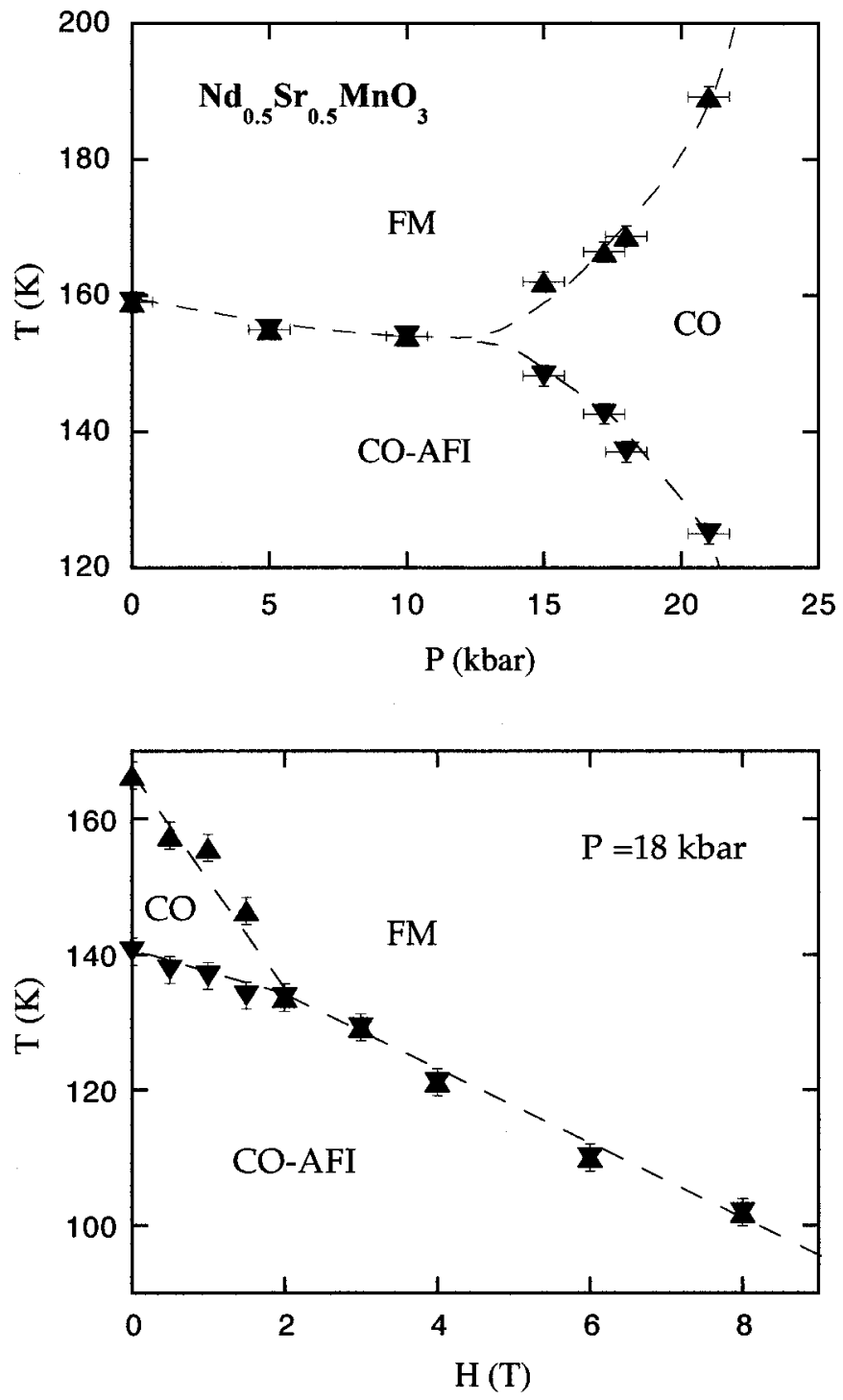

FIG. 5. Phase diagrams on cooling in pressure $P$ and magnetic field $\mathrm{H}$ for $\mathrm{Nd}_{0.5} \mathrm{Sr}_{0.5} \mathrm{MnO}_{3}$. Pressure stabilizes charge order up to high temperature $T$, while depressing antiferromagnetism. Magnetic field drives both transitions to lower $T$, but charge order more quickly, until they merge once again. $\mathrm{FM}=$ ferromagnetic metal; $\mathrm{CO}=$ charge order; $\mathrm{AFI}=$ antiferromagnetic insulator.

the phase diagrams of Fig. 5. A charge order transition intervenes between ferromagnetic metal and antiferromagnetic insulator. This charge ordered state is very robust, moving quickly to higher $T$ with increasing $P$; by $21 \mathrm{kbar}$, the two transitions are $64 \mathrm{~K}$ apart. By contrast, magnetic field suppresses both the charge crystallization and the antiferromagnetism. ${ }^{17}$ The $32 \mathrm{~K}$ difference between the transitions at $P=18 \mathrm{kbar}$ is wiped out by $H=2 \mathrm{~T}$ because of the asymmetric interaction of $H$ with $T_{\mathrm{CO}}$ and $T_{\mathrm{AF}}$.

The different ground states in $\mathrm{Nd}_{1-x} \mathrm{Sr}_{x} \mathrm{MnO}_{3}$ also can be manipulated by varying pressure chemically, e.g., by varying the effective cation radius via substitution of a larger rareearth ion like La. Above a critical La doping, there is a crossover from the charge-exchange (CE)-type antiferromagnetic phase to the $A$-type antiferromagnetic phase, ${ }^{10}$ with 


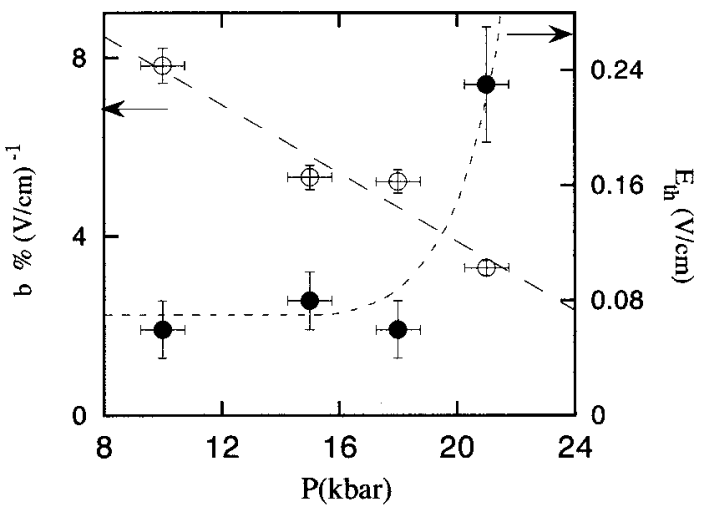

FIG. 6. Variation with pressure $P$ of the threshold field $E_{\mathrm{th}}$ for depinning the charge order (filled circles) and the slope of the nonlinear response $b$ (open circles) at $T=4.2 \mathrm{~K}$.

both phases coexisting ${ }^{14}$ for $x=0.51$. This raises the question as to whether the two features that we observe in the resistivity for $\mathrm{Nd}_{0.5} \mathrm{Sr}_{0.5} \mathrm{MnO}_{3}$ indicate transitions under cooling to the two different antiferromagnetic phases. Such a characterization can be ruled out by the magnetic-field dependence of the two transitions. Moritomo et al. ${ }^{10}$ report that the lower temperature (CE-type) phase is suppressed more by field than the higher temperature (A-type) antiferromagnetic phase. By contrast, we find that the higher temperature transition is far more sensitive to $H$ [Figs. 4 and 5(b)]. Finally, we note that doping with La changes the Curie temperature $\left(T_{C}\right.$ ) by as much as $60 \mathrm{~K}$, while $T_{C}$ changes by less than $5 \mathrm{~K}$ for external pressures $P$ up to 21 kbar. Thus the effects of chemical and applied pressure are not identical, and as such, prevent a direct analogy between the phase diagrams resulting from the two approaches.

Applied pressure also modifies electrical transport in the low-temperature insulator, where the charge ordering gap has opened fully. ${ }^{18}$ The threshold electric field to depin the charge ordered domains is little affected for $P<18 \mathrm{kbar}$, with a moderate rise by our highest pressures (Fig. 6). This corresponds to a reduction in the correlation length from $\xi$ $\approx 4.5 \mu \mathrm{m}$ to $\xi \approx 2.5 \mu \mathrm{m}$. Above $E_{\mathrm{th}}$, where the sliding charge condensate contributes to the conductivity $\sigma$, we fit the percent change in $\sigma$ to a linear dependence on $E: \Delta \sigma / \sigma$ $=a-b E$. From the variation of $b$ in Fig. 6 , we conclude that the fraction of $\sigma$ that can be attributed to the charge ordered condensate (as well as the value of $\sigma$ itself) steadily decrease for $10 \leqslant P \leqslant 21 \mathrm{kbar}$. Any quantitative treatment of the ratio of the nonlinear to the linear conductivity, however, must involve a detailed understanding of the relaxation mechanisms of the different conduction processes present.

In conclusion, we have shown that hydrostatic pressure has the ability to disrupt the careful balance between spin and charge order in a doped perovskite manganite at $50 \%$ hole density. The charge ordered state in $\mathrm{Nd}_{0.5} \mathrm{Sr}_{0.5} \mathrm{MnO}_{3}$ dominates the energetics, with a transition temperature that increases $23 \%$ by $P=21$ kbar. Concomitantly, the antiferromagnetic transition temperature falls 19\%. Magnetic field couples preferentially to the pressure split charge ordered state, and by $H=2 \mathrm{~T}$ the transitions merge and balance is restored. The variation of $T_{\mathrm{CO}}$ and $T_{\mathrm{AF}}$ with $P, H$, and $T$ should fix parameters and restrict possible phase diagrams in Landau-type theories of competing instabilities with (nearly) degenerate energetics. ${ }^{19}$

We are grateful to P. Schiffer for insightful comments and to A. Hoekstra for technical guidance. The work at the University of Chicago was supported by NSF Grant No. DMR9801824. The work at Argonne was supported by the U.S. Department of Energy Materials Science-Office of Science under Contract No. W-31-109-ENG-38.
${ }^{1}$ S. Jin et al., Science 264, 413 (1994).

${ }^{2}$ For a plethora of possibilities, see Y. Tokura et al., J. Appl. Phys. 79, 5288 (1996); A. Moreo, S. Yunoki, and E. Dagotto, Science 283, 2034 (1999).

${ }^{3}$ M. Uehara, S. Mori, C. H. Chen, and S.-W. Cheong, Nature (London) 299, 560 (1999); M. Fäth, S. Freisem, A. A. Menovsky, Y. Tomioka, J. Aarts, and J. A. Mydosh, Science 285, 1540 (1999).

${ }^{4}$ M. Roy, J. F. Mitchell, A. P. Ramirez, and P. Schiffer, Phys. Rev. B 58, 5185 (1998).

${ }^{5}$ S. Yunoki, T. Hotta, and E. Dagotto, Phys. Rev. Lett. 84, 3714 (2000)

${ }^{6}$ H. Kuwahara, Y. Tomioka, A. Asamitsu, Y. Moritomo, and Y. Tokura, Science 270, 961 (1995).

${ }^{7}$ A. J. Millis, P. B. Littlewood, and B. I. Shraiman, Phys. Rev. Lett. 74, 5144 (1995).

${ }^{8}$ See T. F. Rosenbaum, Supercond. Rev. 2, 257 (1998), and references therein.

${ }^{9}$ D. N. Argyriou et al., J. Solid State Chem. 124, 381 (1996).
${ }^{10}$ See also Y. Morimoto, H. Kuwahara, Y. Tomioka, and Y. Tokura, Phys. Rev. B 55, 7549 (1997).

${ }^{11}$ W. Bao, J. D. Axe, S. H. Chen, and S.-W. Cheong, Phys. Rev. Lett. 78, 543 (1997).

${ }^{12}$ H. Fukuyama and P. A. Lee, Phys. Rev. B 17, 535 (1978); P. A. Lee and T. M. Rice, ibid. 19, 3970 (1979).

${ }^{13}$ A. P. Ramirez et al., Phys. Rev. Lett. 76, 3188 (1996).

${ }^{14}$ R. Kajimoto et al., Phys. Rev. B 60, 9506 (1999).

${ }^{15}$ P. M. Woodward, D. E. Cox, T. Vogt, C. N. R. Rao, and A. K. Cheetham, Chem. Mater. 11, 3528 (1999).

${ }^{16}$ A. Husmann, Ph.D. thesis, University of Chicago, 1997.

${ }^{17}$ Superconducting $\mathrm{UPt}_{3}$ has a similar asymmetry in its $P-T$ and $H-T$ phase diagrams. D. S. Jin, S. A. Carter, B. Ellman, T. F. Rosenbaum, and D. G. Hinks, Phys. Rev. Lett. 68, 1597 (1992).

${ }^{18}$ A. Biswas et al., J. Phys.: Condens. Matter 9, L355 (1997).

${ }^{19}$ For (U, Th)Be ${ }_{13}$, see M. Sigrist and T. M. Rice, Phys. Rev. B 39, 2200 (1989); for $\mathrm{UPt}_{3}$ see, e.g., R. Joynt, Supercond. Sci. Technol. 1, 210 (1988); J. A. Sauls, Adv. Phys. 43, 113 (1994). 